\title{
Star-graph expansions for bond-diluted Potts models
}

\author{
Meik Hellmund and Wolfhard Janke周 \\ Institut für Theoretische Physik, Universität Leipzig, \\ Augustusplatz 10/11, D-04109 Leipzig, Germany
}

(Dated: October 28, 2018)

\begin{abstract}
We derive high-temperature series expansions for the free energy and the susceptibility of randombond $q$-state Potts models on hypercubic lattices using a star-graph expansion technique. This method enables the exact calculation of quenched disorder averages for arbitrary uncorrelated coupling distributions. Moreover, we can keep the disorder strength $p$ as well as the dimension $d$ as symbolic parameters. By applying several series analysis techniques to the new series expansions, one can scan large regions of the $(p, d)$ parameter space for any value of $q$. For the bond-diluted 4 -state Potts model in three dimensions, which exhibits a rather strong first-order phase transition in the undiluted case, we present results for the transition temperature and the effective critical exponent $\gamma$ as a function of $p$ as obtained from the analysis of susceptibility series up to order 18. A comparison with recent Monte Carlo data (Chatelain et al., Phys. Rev. E64, 036120(2001)) shows signals for the softening to a second-order transition at finite disorder strength.

PACS numbers:

05.50.+q Lattice theory and statistics (Ising, Potts, etc.)

64.60.Cn Order-disorder transformations; statistical mechanics of model systems

64.60.Fr Equilibrium properties near critical points, critical exponents
\end{abstract}

\section{INTRODUCTION}

Systematic series expansions for statistical models defined on a lattice are a well-known method to study phase transitions and critical phenomena [1]. They provide an useful complement to large-scale numerical simulations, in particular for quenched, disordered systems where the average over many different disorder realizations is numerically very time consuming and only some points in the vast parameter space of the systems can be sampled with realistic effort.

Using high-temperature series expansions, on the other hand, one can obtain for many quantities results which are exact up to a certain order in the inverse temperature. Here the infinite-volume limit can be taken without problems and the quenched disorder is treated exactly. Moreover, one can keep the disorder strength $p$ as well as the dimension $d$ as symbolic parameters and therefore analyse much larger regions of the parameter space of disordered systems. To this end we developed further the method of "star-graph expansion" which allows us to take the disorder average on the level of individual graphs exactly and apply it to $q$-state Potts models with a bimodal quenched distribution of ferromagnetic couplings. Using optimized cluster algorithms for the exact calculation of spin-spin correlators on graphs with arbitrary inhomogeneous couplings, we obtained series up to order 18 in the inverse temperature for the susceptibility and the free energy of bond-diluted Potts models in two, three and four dimensions.

*Electronic address: Meik.Hellmund@itp.uni-leipzig.de;

URL: http://www . physik. uni-leipzig.de/ hellmund

${ }^{\dagger}$ Electronic address: Wolfhard.Janke@itp.uni-leipzig.d
Depending on the dimension $d$ and the number of states $q$, pure Potts models show first- or second-order phase transitions. According to the Harris criterion [2] one expects in the second-order case either the appearance of a new random fixed point $(d=2, q=3,4$ and $d=3, q=2$ ) or logarithmic corrections to the pure fixed point $(d=2, q=2)$. At first-order transitions, randomness softens the transitions. For $d=2$ even infinitesimal disorder induces a continuous transition [3], whereas for $d=3, q>2$ a tricritical point at a finite disorder strength is expected [1]. This softening to a second-order phase transition beyond a tricritical point at some finite disorder strength has recently been verified in Monte Carlo (MC) simulations of the three-dimensional site-diluted 3-state [5] and bond-diluted 4-state [6] Potts model.

The critical part of the series expansion methods lies in the extrapolation techniques which are used in order to obtain information on the critical singularity from a finite number of known coefficients of the high-temperature series. One can question the use of these extrapolation techniques in disordered systems, where the complete singularity structure of the function may be very complicated, involving Griffiths-type singularities or logarithmic corrections [7].

Anyhow, we are able to determine the transition temperature for the bond-diluted 4-state Potts model in three dimensions reliably up to the vicinity of the geometrical percolation point and in good agreement with analytic estimates [8] and MC results [6].

The critical exponent $\gamma$ extracted from our analysis appears to be dependent on the disorder strength which is caused by crossover effects and the complicated singularity structure. Using sophisticated analysis methods, we find a range of the disorder strength where $\gamma_{\mathrm{eff}}=1$, indicating critical behavior governed by a tricritical point.

The rest of the paper is organized as follows. In Sect. II 
we briefly recall the model. In Sect. III we describe the methods used for generating the series, and Sect. IV is devoted to a representation of the analysis techniques used and their application to the study of the bond-diluted 4-state Potts model in three dimensions.

\section{MODEL}

The $q$-state Potts model on the hypercubic lattice $\mathbb{Z}^{d}$, or more generally on any graph $G$ with arbitrary coupling constants $J_{i j}$ assigned to the links $\langle i j\rangle$ of $G$, is defined by its partition function

$$
Z=\sum_{\left\{S_{i}\right\}} \exp \left(\beta \sum_{\langle i j\rangle} J_{i j} \delta\left(S_{i}, S_{j}\right)\right)
$$

where $\beta=1 / k_{B} T$ is the inverse temperature, $S_{i}=$ $1, \ldots, q$ and $\delta(.,$.$) is the Kronecker symbol. In our series$ expansion the combination

$$
v_{i j}=\frac{e^{\beta J_{i j}}-1}{e^{\beta J_{i j}}-1+q}
$$

will be the relevant expansion parameter. In the symmetric high-temperature phase, the susceptibility corresponding to the coupling to an external field $h_{i}$, $\sum_{i} h_{i}\left(\frac{q \delta\left(S_{i}, 1\right)-1}{q-1}\right)$, is given for a graph with $N$ spins by

$$
\chi=\frac{1}{N} \sum_{i} \sum_{j}\left[\left\langle\frac{q \delta\left(S_{i}, S_{j}\right)-1}{q-1}\right\rangle\right] .
$$

Quenched disorder averages [...] are taken over an uncorrelated bimodal distribution of the form

$$
P\left(J_{i j}\right)=(1-p) \delta\left(J_{i j}-J_{0}\right)+p \delta\left(J_{i j}-R J_{0}\right),
$$

which can include spin glasses $(R=-1, p=1 / 2)$, random-bond ferromagnets $(0<R<1)$ and bond dilution $(R=0)$ as special cases. Other distributions can, in principle, also be considered with our method.

\section{SERIES GENERATION}

\section{A. Basic notations from graph theory}

A graph of order $E$ consists of $E$ links connecting $N$ vertices. We consider only connected, undirected graphs that are simple: no link starts and ends at the same vertex and two vertices are never connected by more than one link. Subgraphs are defined by the deletion of links. In this process, isolated vertices can be dropped. A graph of order $E$ has $2^{E}$ (not necessarily non-isomorphic) subgraphs since each link may be present or absent. These subgraphs may consist of several connected components and are called clusters.
An articulation point is a vertex the deletion of which renders the graph disconnected. A graph without articulation points is called "star graph".

A graph is bipartite if the vertices can be separated into red and black vertices so that no link connects two vertices of the same color. Equivalently, all closed paths in the graph consist of an even number of links.

\section{B. Star-graph expansion method}

There are two well-established methods [1] for the systematic generation of high-temperature series expansions, the linked cluster and the star-graph method. The longest known series (up to order $\beta^{25}$ ) for classical spin models without disorder are produced by linked cluster expansions [9]. This technique allows one to obtain series for observables (such as the second moment of the spin-spin correlation function) which have no star-graph expansion. Furthermore, it works with free embeddings of graphs into the lattice which can be counted orders of magnitude faster than the weak embedding numbers used by the star-graph technique. Nonetheless, this method has not yet been applied to problems with quenched disorder.

The star-graph method can be adopted to systems involving quenched disorder [10, 11] since it allows one to take the disorder average on the level of individual graphs. The basic idea is to assemble the value of some extensive thermodynamic quantity $F$ on a large or even infinite graph from its values on subgraphs: Graphs constitute a partially ordered set under the "subgraph" relation. Therefore, for every function $F(G)$ defined on the set of graphs exists another function $W_{F}(G)$ such that for all graphs $G$

$$
F(G)=\sum_{g \subseteq G} W_{F}(g),
$$

and this function can be calculated recursively via

$$
W_{F}(G)=F(G)-\sum_{g \subset G} W_{F}(g) .
$$

This gives for an infinite (e.g. hypercubic) lattice

$$
F\left(\mathbb{Z}^{d}\right)=\sum_{G}\left(G: \mathbb{Z}^{d}\right) W_{F}(G)
$$

where $\left(G: \mathbb{Z}^{d}\right)$ denotes the weak embedding number of the graph $G$ in the given lattice structure 12 .

The following observation makes this a useful method: Let $G$ be a graph with an articulation vertex where two star subgraphs $G_{1,2}$ are glued together. Then $W_{F}(G)$ vanishes if

$$
F(G)=F\left(G_{1}\right)+F\left(G_{2}\right)
$$


TABLE I: Number of star graphs with $E$ links and nonvanishing embedding numbers on $\mathbb{Z}^{d}$.

\begin{tabular}{l|r|r|r|r|r|r|r|r|r|r|r|r|r|r|r|r}
\hline \hline order $E$ & 1 & 4 & 6 & 7 & 8 & 9 & 10 & 11 & 12 & 13 & 14 & 15 & 16 & 17 & 18 & 19 \\
\hline$\#$ & 1 & 1 & 1 & 1 & 2 & 3 & 8 & 9 & 29 & 51 & 142 & 330 & 951 & 2561 & 7688 & 23078 \\
\hline \hline
\end{tabular}

An observable $F$ for which Eq. (8) is true on arbitrary graphs with articulation points allows a star-graph expansion. All non-star graphs have zero weight $W_{F}$ in the sum Eq. (7).

It is easy to see that the (properly normalized) free energy $\log Z$ has this property and it can be proved 10 that the inverse susceptibility $1 / \chi$ has it, too, even for arbitrary inhomogeneous couplings $J_{i j}$. This restricts the sum in Eq. (7) to a sum over star graphs. The linearity of Eqs. (5)-(7) enables the calculation of quenched averages over the coupling distribution on the level of individual graphs.

The resulting recipe for the susceptibility series is:

- Graph generation and embedding number counting.

- Calculation of $Z(G)$ and the correlation matrix $M_{n m}(G)=\operatorname{Tr}\left(q \delta\left(S_{n}, S_{m}\right)-1\right) e^{-\beta H\left(\left\{J_{i j}\right\}\right)}$ for all graphs as polynomials in $E$ variables $v_{i j}$.

- Inversion of the $Z$ polynomial as a series up to the desired order.

- Averaging over quenched disorder, $N_{n m}(G)=\left[M_{n m} / Z\right]_{P(J)}$, resulting in a matrix of polynomials in $(p, v)$.

- Inversion of the matrix $N_{n m}$ and subgraph subtraction, $W_{\chi}(G)=\sum_{n, m}\left(N^{-1}\right)_{n m}-\sum_{g \subset G} W_{\chi}(g)$.

- Collecting the results from all graphs, $1 / \chi=\sum_{G}\left(G: \mathbb{Z}^{d}\right) W_{\chi}(G)$.

\section{Generation of star graphs and calculation of embedding numbers}

The most complicated part in every attempt to generate lists of graphs by recursively adding nodes and edges to a smaller list is the isomorphism test, i.e., the decision whether two given adjacency lists or adjacency matrices describe the same graph modulo relabelling and reordering of edges and nodes. We used the nauty package by McKay [13] which makes very fast isomorphism tests by calculating a canonical representation of the automorphism group of the graphs.

Since we are only interested in star graphs with nonvanishing weak embedding numbers in $\mathbb{Z}^{d}$, the following simple observations are helpful:

- Only bipartite graphs occur since $\mathbb{Z}^{d}$ is bipartite.

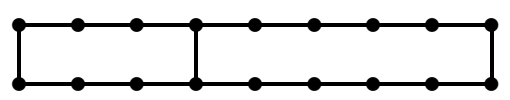

$$
\begin{aligned}
& 7620\left(\begin{array}{l}
d \\
2
\end{array}\right)+76851600\left(\begin{array}{l}
d \\
3
\end{array}\right)+14650620864\left(\begin{array}{l}
d \\
4
\end{array}\right) \\
& +404500471680\left(\begin{array}{l}
d \\
5
\end{array}\right)+3355519311360\left(\begin{array}{l}
d \\
6
\end{array}\right)
\end{aligned}
$$

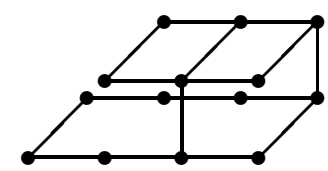

$$
12048\left(\begin{array}{l}
d \\
3
\end{array}\right)+396672\left(\begin{array}{l}
d \\
4
\end{array}\right)+2127360\left(\begin{array}{l}
d \\
5
\end{array}\right)+2488320\left(\begin{array}{l}
d \\
6
\end{array}\right)
$$

FIG. 1: Two star graphs of order 17 and 19 and their weak embedding numbers up to 6 dimensions.

- A generic $k$-dimensional embedding (i.e. one which really needs all $k$ dimensions) contributes in $d>k$ dimensions with degeneracy $\left(\begin{array}{l}d \\ k\end{array}\right)$.

- A biconnected graph of odd order $E=2 n+1$ has generic embeddings only up to dimension $n$ since it must have at least two edges in each dimension.

- The only biconnected graph of even order $E=2 n$ which has generic embeddings of dimension $n$ is the cycle of length $2 n$. All the other graphs will use at most $n-1$ dimensions.

For the embedding count we implemented a refined version of the backtracing algorithm by Martin [12]. We did extensive tests to find the optimal algorithm for the "innermost" loop, the test for collisions in the embedding, and ended up using optimized hash tables.

By this means, we classified for the first time all star graphs up to order 19 which can be embedded in hypercubic lattices (see Table [i]) and calculated their (weak) embedding numbers for $d$-dimensional hypercubic lattices (up to order 17 for arbitrary $d$, order 18 and 19 for dimensions $\leq 4)$, see Fig. 目 for typical results.

\section{Cluster representation}

The partition function and the matrix of correlations $M_{n m}$ for each graph are calculated with arbitrary symbolic couplings $J_{i j}$ using the cluster representation

$$
\begin{aligned}
Z & =q^{N-E} \prod_{\langle i j\rangle}\left(e^{\beta J_{i j}}-1+q\right) \mathcal{Z}, \\
\mathcal{Z} & =q^{-N} \operatorname{Tr} \prod_{\langle i j\rangle}\left[1-v_{i j}+v_{i j} q \delta\left(S_{i}, S_{j}\right)\right] \\
& =\sum_{C} q^{e+c-N}\left(\prod_{\langle i j\rangle \in C} v_{i j}\right)\left(\prod_{\langle i j\rangle \notin C}\left(1-v_{i j}\right)\right) .
\end{aligned}
$$


Here the sum goes over all clusters $C \subseteq G, E$ is the number of links (= order) of the graph $G, e$ is the number of links of the cluster and $c$ the number of connected components of $C$. $\mathcal{Z}$ is normalized such that $\log \mathcal{Z}$ has a star-graph expansion. This essentially reduces the partition sum from a sum over $q^{N}$ states to a sum over $2^{E}$ clusters. In the Ising case $q=2$ another huge simplification takes place since only clusters where all vertices are of even degree contribute to the cluster sum.

The $2^{E}$ clusters belonging to a graph are enumerated by Gray codes [14 such that two consecutive clusters in the sum (11) differ by exactly one (added or deleted) link. Gray codes are a reordering of the binary representation of numbers such that the difference to the successor is in exactly one bit position. For example, for $E=4$ the sequence is $0000,0001,0011,0010,0110,0111,0101,0100$, $1100,1101,1111,1110,1010,1011,1001,1000$ where zeros denote the deleted links. This allows to speed up the calculation considerably by re-using every term in the sum for the calculation of the next one.

The calculation of the susceptibility involves the matrix of correlations $M_{n m}$. The effect of inserting $\frac{q \delta\left(S_{i}, S_{j}\right)-1}{q-1}$ into the trace of Eq. (10) can easily be seen: we get one if the vertices $n$ and $m$ belong to the same connected component of the cluster and zero otherwise. Therefore,

$$
M_{n m} \propto \sum_{C_{n m}} q^{e+c-N}\left(\prod_{\langle i j\rangle \in C} v_{i j}\right)\left(\prod_{\langle i j\rangle \notin C}\left(1-v_{i j}\right)\right),
$$

where the sum is restricted to all clusters $C_{n m} \subseteq G$ in which the vertices $n$ and $m$ are connected.

For the symbolic calculations we developed a $\mathrm{C}^{++}$ template library using an expanded degree-sparse representation of polynomials and series in many variables. The open source library GMP is used for the arbitraryprecision arithmetics.

Our longest series, up to order 18, are obtained for the case of bond dilution where (4) simplifies to

$$
P\left(J_{i j}\right)=(1-p) \delta\left(J_{i j}-J_{0}\right)+p \delta\left(J_{i j}\right),
$$

since in this case the disorder average for a series is most easily done via

$$
\left[v_{1}^{n_{1}} \ldots v_{k}^{n_{k}}\right]_{P(J)}=(1-p)^{k} v_{0}^{n_{1}+\ldots+n_{k}} .
$$

\section{SERIES ANALYSIS: TECHNIQUES AND RESULTS}

In the following we shall illustrate the analysis using the bond-diluted 4-state Potts model in three dimensions as our primary example. This model exhibits in the pure case a strong first-order transition which is expected to stay first order up to some finite disorder strength, before

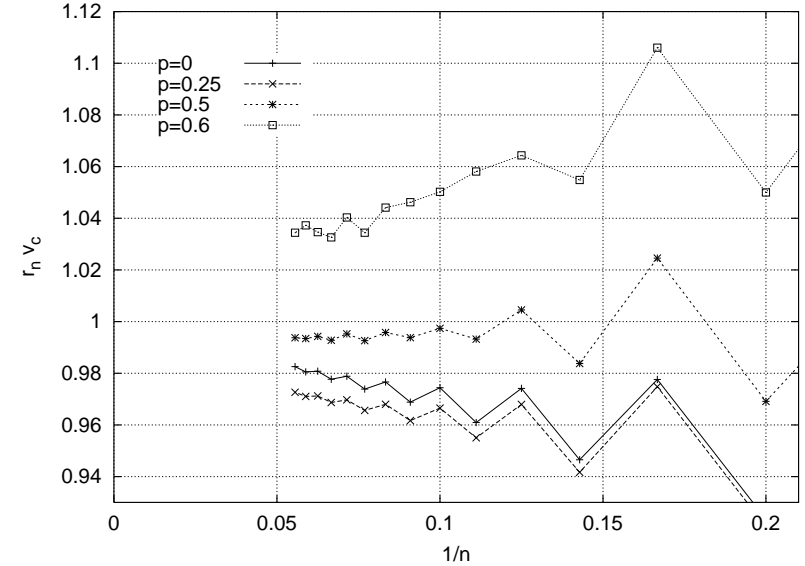

FIG. 2: Ratio approximants for different dilutions $p$ vs. $1 / n$.

it gets softened to a second-order transition governed by a disordered fixed point.

In the latter case we are interested in locating powerlaw divergences in the susceptibility series of the form

$$
\chi(v)=A\left(v_{c}-v\right)^{-\gamma}+\ldots
$$

For such a critical behavior many different series analysis techniques have been discussed in the literature which all have their merits and drawbacks [15].

To localize a first-order transition point, however, a high-temperature series alone is not sufficient since there the correlation length remains finite and no critical singularity occurs. In analysing series by ratio, Padé or differential approximants, the approximant will provide an analytic continuation of the thermodynamic quantities beyond the transition point into a metastable region on a pseudo-spinodal line with a singularity $T_{c}^{*}<T_{c}$ and effective "critical exponents" at $T_{c}^{*}$.

Employing the techniques described above we obtained the high-temperature series expansions for the susceptibility up to order 18 with coefficients given as polynomials in the disorder strength $p$, as listed in the Appendix for general dimensions $d \leq 4$. For such a series in two variables, the method of partial differential approximants [16] should be well suited. Up to date, however, the only application of this method to a tricritical point 17] used a test series of order 50 generated from an exactly solvable model. In our case, it was unable to give conclusive results. Therefore, we confined ourselves to the analysis of single-parameter series for selected values of $p$.

The ratio method is the least sophisticated method of series analysis, but usually it is quite robust and gives a good first estimate of the series behavior. It assumes that the expected singularity of the form (15) is the closest to the origin. Then the consecutive ratios of series coefficients behave asymptotically as

$$
r_{n}=\frac{a_{n}}{a_{n-1}}=v_{c}^{-1}\left(1+\frac{\gamma-1}{n}\right) .
$$




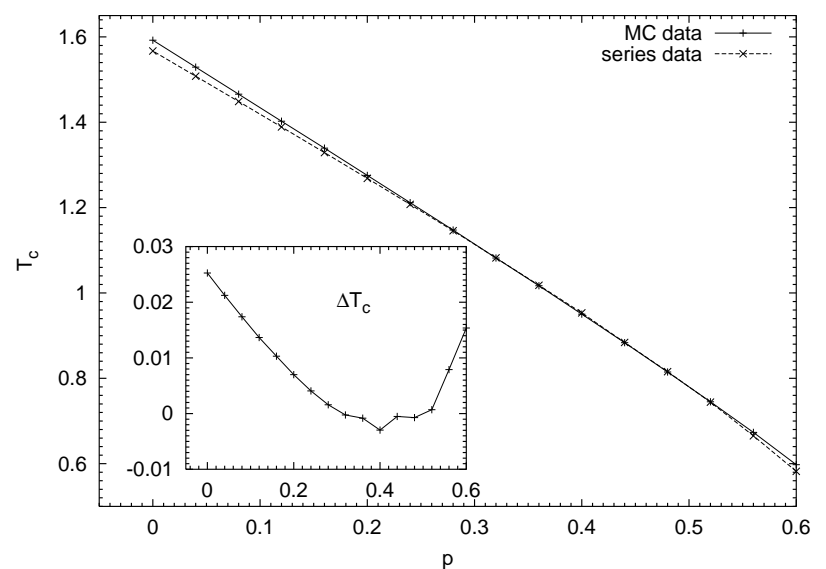

FIG. 3: Critical temperature for different dilution $p$ as obtained from MC simulations $[6]$ and DLog-Padé series analyses. The inset shows the difference between the two estimates.

Figure 2 shows these ratios for different values of $p$. In order to make them visually comparable, they are normalized by their respective critical couplings $v_{c}$. For small $p$ they show the typical oscillations related to the existence of an antiferromagnetic singularity at $-v_{c}$. Near the percolation threshold at $p=0.751188$ 18 (where $T_{c}$ goes to 0$)$ the series is clearly ill-behaved, related to the $\exp (1 / T)$ singularity expected there. Besides that, we observe that the slope $(\propto \gamma-1)$ is increasing with $p$, changing from $\gamma<1$ to $\gamma>1$ around $p=0.5$.

The widely used DLog-Padé method consists in calculating Padé approximants to the logarithmic derivative of $\chi(v)$. The smallest real pole of the approximant is an estimation of $v_{c}$ and its residue gives $\gamma$. Figure 3 compares the critical temperature, estimated from an average of 25-30 Padé approximants for each value of $p$ 22], with the results of recent MC simulations [6]. For small $p$, in the first-order region, the series underestimates the critical temperature. As explained above, this is an estimate not of $T_{c}$ but of $T_{c}^{*}$. Between $p=0.3$ and $p=0.5$, the estimates confirm, within errors, the MC results, indicating that now both methods see the same second-order transition. Beyond $p=0.5$, the scatter of different Padé approximants increases rapidly, related to the crossover to the percolation point.

The situation is more complicated with respect to the critical exponent $\gamma$. A DLog-Padé analysis gives inconclusive results due to a large scattering between different Padé approximants, as shown in Fig. 4. One possible reason for this failure is the existence of confluent singularities: The dots in Eq. (15) indicate correction terms which can be parametrized as follows:

$\chi(v)=A\left(v_{c}-v\right)^{-\gamma}\left[1+A_{1}\left(v_{c}-v\right)^{\Delta_{1}}+A_{2}\left(v_{c}-v\right)^{\Delta_{2}}+\ldots\right]$

where $\Delta_{i}$ are the confluent correction exponents. We used different more sophisticated analysis methods, such as inhomogeneous differential approximants [15] and the

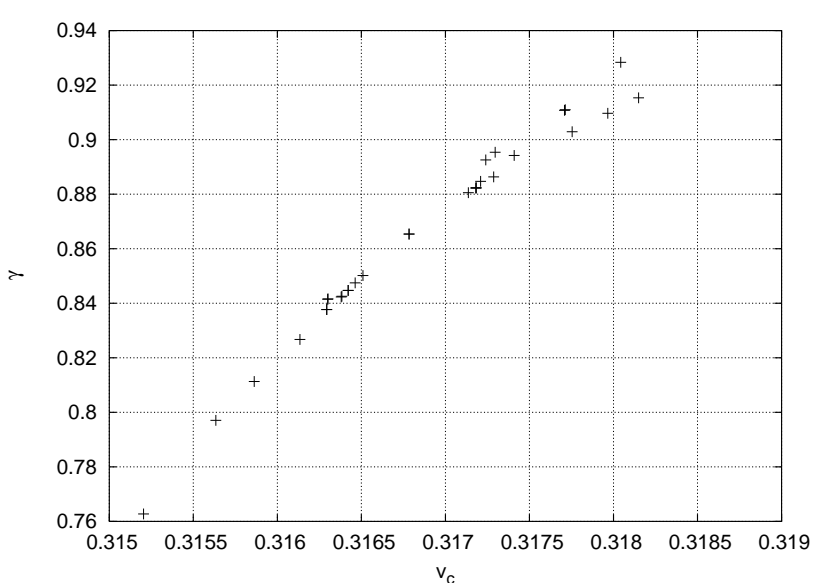

FIG. 4: Scattering of different Padé approximants at a dilution $p=0.4$ : critical exponent $\gamma$ against critical coupling $v_{c}$.

methods M1 and M2 [19], especially tailored to deal with confluent singularities. In the case at hand, the BakerHunter method 20] appeared to be quite successful, giving consistent results at larger dilutions $p>0.35$ where the DLog-Padé analysis failed. Assume the function under investigation has confluent singularities

$$
F(z)=\sum_{i=1}^{N} A_{i}\left(1-\frac{z}{z_{c}}\right)^{-\lambda_{i}}=\sum_{n=0} a_{n} z^{n} .
$$

This can be transformed into an auxiliary function $g(t)$ which is meromorphic and therefore suitable for Padé approximation. After the substitution $z=z_{c}\left(1-e^{-t}\right)$ we expand $F(z(t))=\sum_{n} c_{n} t^{n}$ and construct the new series

$$
g(t)=\sum_{n=0} n ! c_{n} t^{n}=\sum_{i=1}^{N} \frac{A_{i}}{1-\lambda_{i} t} .
$$

We see that Padé approximants to $g(t)$ have poles at $t=1 / \lambda_{i}$, with residues at the poles of $-A_{i} / \lambda_{i}$. This method is applied by plotting these poles and residues for different Padé approximants to $g(t)$ as functions of $z_{c}$. The optimal set of values for the parameters is determined visually from the best clustering of different Padé approximants, as demonstrated in Fig. 5 .

Using this method, our results for the critical exponent $\gamma$ are plotted in Fig. 6. They show an effective exponent monotonically increasing with $p$ but reaching a plateau at $\gamma=1$ for dilutions between $p=0.42$ and $p=0.46$. The following sharp increase is to be interpreted as due to the crossover to the percolation fixed point $p_{c}=0.751188$, $T_{c}=0$, where a $\chi \sim \exp (1 / T)$ behavior is expected.

, It is well known (see, e.g., Ref. 21]) that series analysis in crossover situations is extremely difficult. If the parameter $p$ interpolates between regions governed by different fixed points, the exponent obtained from a finite number of terms of a series expansion must cross somehow between its universal values, and does this usually 

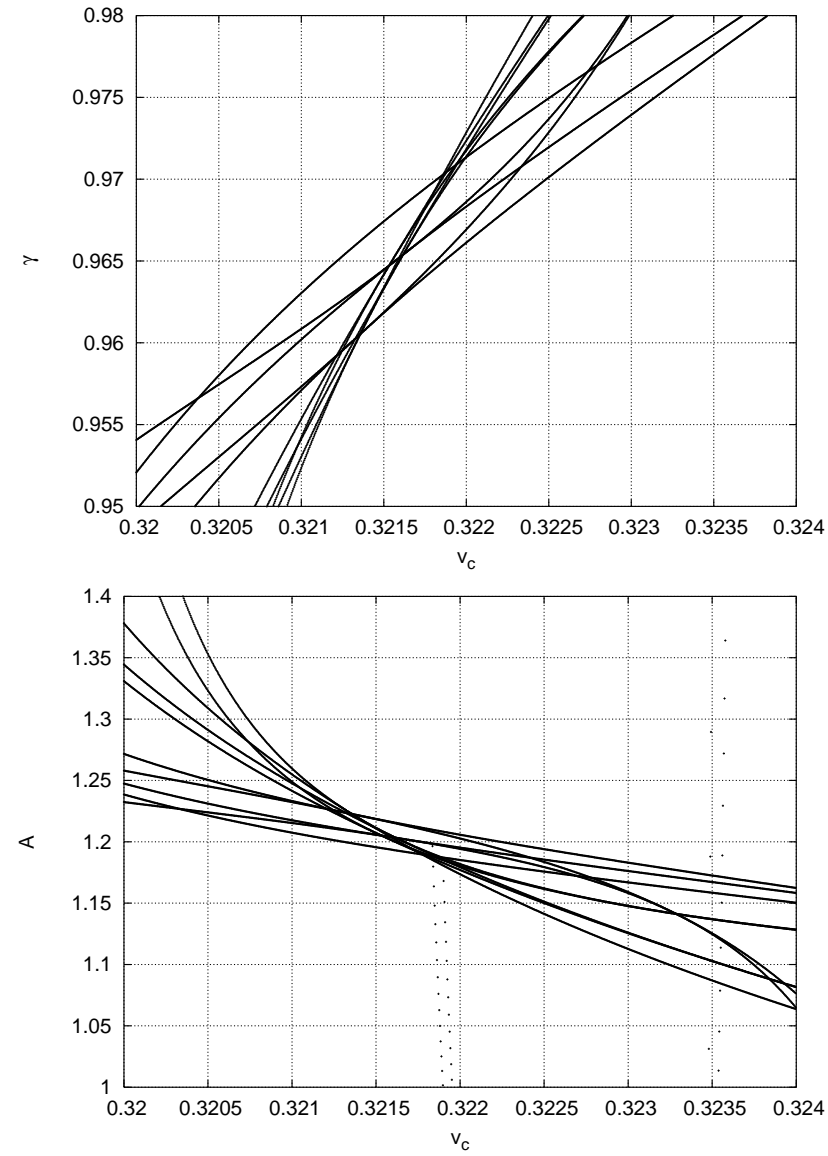

FIG. 5: Values for the critical exponent $\gamma$ and amplitude $A$ at $p=0.4$ as function of trial $v_{c}$ estimates from the Baker-Hunter analysis. From the clustering of different Padé approximants in both pictures we estimate $v_{c}=0.3217, \gamma=0.966$, and $A=1.21$.

quite slowly. Therefore it does not come as a surprise that the MC simulations quoted above see the onset of a second order phase transition already for smaller values of the disorder strength $p$. The mere existence of a plateau in $\gamma_{\text {eff }}(p)$, however, is an indication that here truly critical behavior is seen. It is governed by a fixed point for which we obtain $\gamma=1.00(3)$. Here, as always in series analyses, the error estimates the scattering of different approximants.

\section{CONCLUSIONS}

We have implemented a comprehensive toolbox for generating and enumerating star graphs as required for high-temperature series expansions of quenched, disordered systems. Monte Carlo simulations of systems with quenched disorder require an enormous amount of computing time because many realizations have to be simulated for the quenched average. For this reason it is hardly possible to scan a whole parameter range. Using high-temperature series expansions, on the other hand,

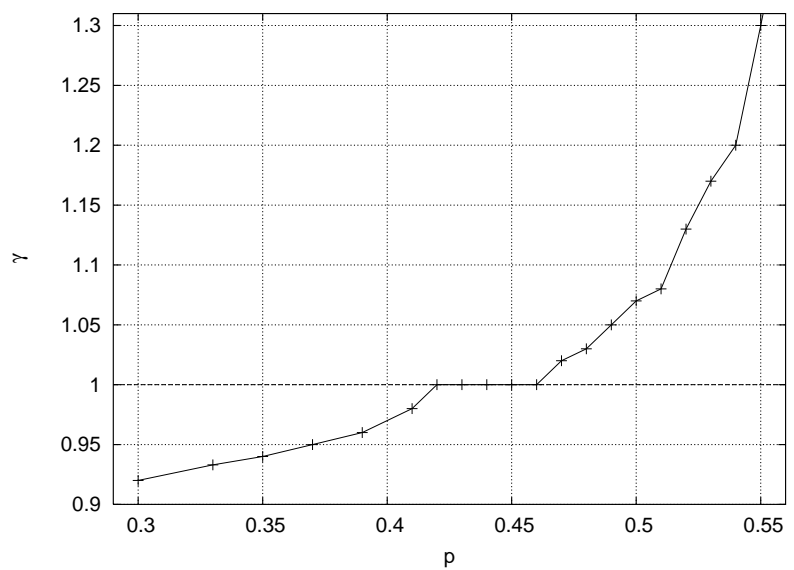

FIG. 6: Effective critical exponent $\gamma$ as function of the dilution $p$ from Baker-Hunter analyses.

one can obtain this average exactly. Since the relevant parameters (degree of disorder $p$, spatial dimension $d$, number of states $q$, etc.) can be kept as symbolic variables, the number of potential applications is very large.

Here we presented an analysis of the three-dimensional bond-diluted 4-state Potts model. The phase diagram confirms recent Monte Carlo results and, by comparing with the numerical data, we also see signals for the onset of a second-order transition at a finite disorder strength.

\section{Acknowledgments}

Support by DFG grant No. JA 483/17-1 and partial support from the German-Israel-Foundation under grant No. I-653-181.14/1999 is gratefully acknowledged. 


\section{APPENDIX A}

As an example, we publish here the inverse susceptibility for the bond-diluted 4-state Potts model in $d \leq 4$ dimensions up to order $v^{18}(P=1-p)$ :

$$
\begin{aligned}
& \chi^{-1}(P, v, d)=1-2 P v d+2 P^{2} v^{2} d-2 P^{3} v^{3} d+\left[2 P^{4} d-16 P^{4}\left(\begin{array}{l}
d \\
2
\end{array}\right)\right] v^{4}+\left[-2 P^{5} d+\left(24 P^{4}+72 P^{5}\right)\left(\begin{array}{l}
d \\
2
\end{array}\right)\right] v^{5} \\
& +\left[2 P^{6} d+\left(24 P^{4}-96 P^{5}-248 P^{6}\right)\left(\begin{array}{l}
d \\
2
\end{array}\right)-768 P^{6}\left(\begin{array}{l}
d \\
3
\end{array}\right)\right] v^{6} \\
& +\left[-2 P^{7} d+\left(24 P^{4}-96 P^{5}+264 P^{6}+640 P^{7}\right)\left(\begin{array}{l}
d \\
2
\end{array}\right)+\left(576 P^{6}+3264 P^{7}\right)\left(\begin{array}{l}
d \\
3
\end{array}\right)\right] v^{7} \\
& +\left[2 P^{8} d+\left(72 P^{4}-96 P^{5}+264 P^{6}-216 P^{7}-1384 P^{8}\right)\left(\begin{array}{l}
d \\
2
\end{array}\right)+\left(576 P^{6}-144 P^{7}-22704 P^{8}\right)\left(\begin{array}{l}
d \\
3
\end{array}\right)-62208 P^{8}\left(\begin{array}{l}
d \\
4
\end{array}\right)\right] v^{8} \\
& +\left[-2 P^{9} d+\left(-72 P^{4}-288 P^{5}+264 P^{6}-312 P^{7}-1416 P^{8}+1888 P^{9}\right)\left(\begin{array}{l}
d \\
2
\end{array}\right)\right. \\
& \left.+\left(576 P^{6}-720 P^{7}+720 P^{8}+66944 P^{9}\right)\left(\begin{array}{l}
d \\
3
\end{array}\right)+\left(31104 P^{8}+221312 P^{9}\right)\left(\begin{array}{l}
d \\
4
\end{array}\right)\right] v^{9} \\
& +\left[2 P^{10} d+\left(-72 P^{4}+288 P^{5}+648 P^{6}+384 P^{7}-144 P^{8}+9336 P^{9}-296 P^{10}\right)\left(\begin{array}{l}
d \\
2
\end{array}\right)\right. \\
& \left.+\left(576 P^{6}+3456 P^{7}+19296 P^{8}+75456 P^{9}-387168 P^{10}\right)\left(\begin{array}{l}
d \\
3
\end{array}\right)+\left(31104 P^{8}+109440 P^{9}-4000512 P^{10}\right)\left(\begin{array}{l}
d \\
4
\end{array}\right)\right] v^{10} \\
& +\left[-2 P^{11} d+\left(-72 P^{4}+288 P^{5}-504 P^{6}-1008 P^{7}-3024 P^{8}+3144 P^{9}-33336 P^{10}-9616 P^{11}\right)\left(\begin{array}{c}
d \\
2
\end{array}\right)\right. \\
& +\left(576 P^{6}-1440 P^{7}-8352 P^{8}-31248 P^{9}-309744 P^{10}+781824 P^{11}\right)\left(\begin{array}{l}
d \\
3
\end{array}\right) \\
& \left.+\left(31104 P^{8}+11520 P^{9}+635520 P^{10}+10415872 P^{11}\right)\left(\begin{array}{l}
d \\
4
\end{array}\right)\right] v^{11} \\
& +\left[2 P^{12} d+\left(-216 P^{4}+288 P^{5}-216 P^{6}-4272 P^{7}+240 P^{8}+11856 P^{9}-4968 P^{10}+81744 P^{11}+37320 P^{12}\right)\left(\begin{array}{l}
d \\
2
\end{array}\right)\right. \\
& +\left(2880 P^{6}-31392 P^{7}+14112 P^{8}+169200 P^{9}+489024 P^{10}+2692800 P^{11}-5811664 P^{12}\right)\left(\begin{array}{l}
d \\
3
\end{array}\right) \\
& \left.+\left(31104 P^{8}+273024 P^{9}+3204864 P^{10}+16037760 P^{11}-179275648 P^{12}\right)\left(\begin{array}{l}
d \\
4
\end{array}\right)\right] v^{12} \\
& +\left[-2 P^{13} d+\left(216 P^{4}+864 P^{5}-792 P^{6}-3912 P^{7}+29736 P^{8}+5952 P^{9}-20736 P^{10}+23088 P^{11}\right.\right. \\
& \left.-144624 P^{12}-96160 P^{13}\right)\left(\begin{array}{l}
d \\
2
\end{array}\right) \\
& +\left(-1728 P^{6}-31536 P^{7}+162288 P^{8}+15408 P^{9}-223344 P^{10}-113760 P^{11}-8412192 P^{12}+5990784 P^{13}\right)\left(\begin{array}{l}
d \\
3
\end{array}\right) \\
& \left.+\left(31104 P^{8}+67968 P^{9}+1022976 P^{10}-693504 P^{11}-16255872 P^{12}+304010112 P^{13}\right)\left(\begin{array}{l}
d \\
4
\end{array}\right)\right] v^{13} \\
& +\left[2 P^{14} d+\left(216 P^{4}-864 P^{5}-1944 P^{6}-8616 P^{7}+18360 P^{8}-99600 P^{9}-65544 P^{10}+33936 P^{11}\right.\right. \\
& \left.-86952 P^{12}+73704 P^{13}+169400 P^{14}\right)\left(\begin{array}{l}
d \\
2
\end{array}\right) \\
& +\left(-1728 P^{6}-55152 P^{7}+67248 P^{8}-894240 P^{9}-918000 P^{10}+2799648 P^{11}+8589744 P^{12}\right. \\
& \left.+58983984 P^{13}-98045424 P^{14}\right)\left(\begin{array}{l}
d \\
3
\end{array}\right) \\
& \left.+\left(31104 P^{8}-1057536 P^{9}+17280 P^{10}+24870528 P^{11}+179980416 P^{12}+1095494784 P^{13}-7487817088 P^{14}\right)\left(\begin{array}{l}
d \\
4
\end{array}\right)\right] v^{14} \\
& +\left[-2 P^{15} d+\left(216 P^{4}-864 P^{5}+1512 P^{6}-4536 P^{7}+44568 P^{8}-55200 P^{9}+168480 P^{10}+363072 P^{11}-11832 P^{12}\right.\right. \\
& \left.+530040 P^{13}+501600 P^{14}-145632 P^{15}\right)\left(\begin{array}{l}
d \\
2
\end{array}\right) \\
& +\left(-1728 P^{6}-41040 P^{7}+209232 P^{8}-609984 P^{9}+1319328 P^{10}+7874208 P^{11}+5670048 P^{12}+24319296 P^{13}\right. \\
& \left.-141840288 P^{14}-14817536 P^{15}\right)\left(\begin{array}{l}
d \\
3
\end{array}\right) \\
& +\left(31104 P^{8}-740736 P^{9}+131328 P^{10}+22334976 P^{11}+66366720 P^{12}+188319744 P^{13}\right. \\
& \left.\left.-1467511296 P^{14}+5362518016 P^{15}\right)\left(\begin{array}{l}
d \\
4
\end{array}\right)\right] v^{15} \\
& +\left[2 P^{16} d+\left(648 P^{4}-864 P^{5}+1512 P^{6}+24336 P^{7}+38496 P^{8}-148008 P^{9}+1656 P^{10}+117024 P^{11}-1325376 P^{12}\right.\right. \\
& \left.+7200 P^{13}-1644000 P^{14}-2926176 P^{15}-373984 P^{16}\right)\left(\begin{array}{l}
d \\
2
\end{array}\right) \\
& +\left(-1728 P^{6}+163296 P^{7}+105984 P^{8}-2305728 P^{9}-1857888 P^{10}-6620544 P^{11}-48148992 P^{12}+18163728 P^{13}\right. \\
& \left.+118520640 P^{14}+1144225008 P^{15}-1918717248 P^{16}\right)\left(\begin{array}{l}
d \\
3
\end{array}\right) \\
& +\left(217728 P^{8}-3438720 P^{9}-7119360 P^{10}-64137600 P^{11}-149601024 P^{12}+1152714240 P^{13}+8368094208 P^{14}\right. \\
& \left.\left.+58294742400 P^{15}-317165909504 P^{16}\right)\left(\begin{array}{l}
d \\
4
\end{array}\right)\right] v^{16} \\
& +\left[-2 P^{17} d+\left(-648 P^{4}-2592 P^{5}+1512 P^{6}+15408 P^{7}-161712 P^{8}-123384 P^{9}+66792 P^{10}+39264 P^{11}\right.\right. \\
& \left.-1976760 P^{12}+3413424 P^{13}+848256 P^{14}+4241568 P^{15}+8541960 P^{16}+2398960 P^{17}\right)\left(\begin{array}{l}
d \\
2
\end{array}\right) \\
& +\left(-1728 P^{6}+109728 P^{7}-984096 P^{8}-1584432 P^{9}+413424 P^{10}-11287296 P^{11}-32069376 P^{12}+206240976 P^{13}\right. \\
& \left.+380730960 P^{14}+1087235856 P^{15}-1859056704 P^{16}-2643006384 P^{17}\right)\left(\begin{array}{l}
d \\
3
\end{array}\right) \\
& +\left(-93312 P^{8}-2522880 P^{9}-4468608 P^{10}-63930240 P^{11}-127255680 P^{12}+1693209600 P^{13}+6161021568 P^{14}\right. \\
& \left.\left.+23385824256 P^{15}-50368269312 P^{16}-105383991680 P^{17}\right)\left(\begin{array}{l}
d \\
4
\end{array}\right)\right] v^{17}
\end{aligned}
$$




$$
\begin{aligned}
+ & {\left[2 P^{18} d+\left(-648 P^{4}+2592 P^{5}+4104 P^{6}+83520 P^{7}-82080 P^{8}+465984 P^{9}+586080 P^{10}+605064 P^{11}-166248 P^{12}\right.\right.} \\
& \left.+8121312 P^{13}-4714536 P^{14}-2886168 P^{15}-3604536 P^{16}-20651832 P^{17}-7297424 P^{18}\right)\left(\begin{array}{l}
d \\
2
\end{array}\right) \\
& +\left(-8640 P^{6}+518400 P^{7}-492480 P^{8}+3752592 P^{9}+12513744 P^{10}-23522256 P^{11}-66704640 P^{12}-53106912 P^{13}\right. \\
& \left.-884626272 P^{14}-122168448 P^{15}+2205877392 P^{16}+22700601216 P^{17}-42014019168 P^{18}\right)\left(\begin{array}{c}
d \\
3
\end{array}\right) \\
& +\left(-93312 P^{8}+657024 P^{9}+23180544 P^{10}-169350912 P^{11}-762268032 P^{12}-3977024256 P^{13}-10126195200 P^{14}\right. \\
& \left.\left.+47139877632 P^{15}+379559824128 P^{16}+2866361546496 P^{17}+3747410465664 P^{18}\right)\left(\begin{array}{c}
d \\
4
\end{array}\right)\right] v^{18}
\end{aligned}
$$

[1] C. Domb and M. S. Green, eds., Series Expansions for Lattice Models, vol. 3 of Phase Transitions and Critical Phenomena (Academic Press, New York, 1974).

[2] A. B. Harris, Journal of Physics C: Solid State Physics 7, 1671 (1974).

[3] M. Aizenman and J. Wehr, Phys. Rev. Lett. 62, 2503 (1989).

[4] J. Cardy and J. L. Jacobsen, Phys. Rev. Lett. 79, 4063 (1997).

[5] H. G. Ballesteros, L. A. Fernández, V. Martín-Mayor, A. Muñoz Sudupe, G. Parisi, and J. J. Ruiz-Lorenzo, Phys. Rev. B 61, 3215 (2000).

[6] C. Chatelain, B. Berche, W. Janke, and P. E. Berche, Phys. Rev. E 64, 036120 (2001).

[7] J. Cardy, preprint cond-mat/9911024.

[8] L. Turban, Journal of Physics C: Solid State Physics 13, L13 (1980).

[9] P. Butera and M. Comi, preprint hep-lat/0204007.

[10] R. R. P. Singh and S. Chakravarty, Phys. Rev. B 36, 546 (1987).

[11] D. C. Rapaport, Journal of Physics C: Solid State Physics 5, 1830, 2813 (1972).

[12] J. L. Martin, in [1], pp. 97-112.
[13] B. D. McKay, Congressus Numerantium 30, 45 (1981), http://cs.anu.edu.au/ bdm/nauty/.

[14] W. H. Press, S. A. Teukolsky, W. T. Vetterling, and B. P. Flannery, Numerical Recipes in $C$ (Cambridge University Press, Cambridge, 1992).

[15] A. J. Guttmann, in vol. 13 of Phase Transitions and Critical Phenomena, edited by C. Domb and J. L. Lebowitz, (Academic Press, New York, 1989), pp. 1-234.

[16] M. E. Fisher and J.-H. Chen, in Phase Transitions: Cargèse 1980, edited by M. Lévy, J. C. Le Guillou, and J. Zinn-Justin (Plenum, New York, 1982), pp. 169-216.

[17] Z. Salman and J. Adler, Journal of Physics A: Mathematical and General 30, 1979 (1997).

[18] C. D. Lorenz and R. M. Ziff, Phys. Rev. E 57, 230 (1998).

[19] L. Klein, J. Adler, A. Aharony, A. B. Harris, and Y. Meir, Phys. Rev. B 43, 11249 (1991).

[20] G. A. Baker and D. L. Hunter, Phys. Rev. B 7, 3377 (1973).

[21] M. Barma and M. E. Fisher, Phys. Rev. B 31, 5954 (1985).

[22] Notice that " $p$ " in the present notation corresponds to " $1-p$ " in Ref. [6]. 